\title{
Expression of orotate phosphoribosyl transferase in human pancreatic cancer: Implication for the efficacy of uracil and tegafur-based adjuvant chemotherapy
}

\author{
YOSHINORI NIO ${ }^{1,4}$, TOMOKO TOGA ${ }^{1}$, RIRUKE MARUYAMA $^{2}$ and MASAKAZU FUKUSHIMA ${ }^{3}$ \\ ${ }^{1}$ First Department of Surgery, and ${ }^{2}$ Clinical Pathology, Shimane University School of Medicine, \\ Izumo; ${ }^{3}$ Tokushima Research Center, TAIHO Pharmaceutical Co., Ltd., Tokushima, Japan
}

Received December 18, 2006; Accepted March 16, 2007

\begin{abstract}
The enzyme orotate phosphoribosyl transferase (OPRT) is involved in the metabolism of the anticancer drug 5-fluorouracil (5-FU), and is a key enzyme for conversion of 5-FU to its active form in tumor tissue. Little is known regarding the significance of OPRT in human pancreatic cancer. The present study was designed to assess the association between the activity of OPRT in the tumor, and the clinicopathological status and prognosis of human resectable pancreatic cancer, especially regarding its relevance to the efficacy of adjuvant chemotherapy with uracil and tegafur (UFT), cyclophosphamide (CPA) and/or gemcitabine (GEM). The present study included 99 resectable pancreatic cancers, which were all invasive ductal tubular carcinomas. OPRT was immunostained with a rabbit anti-human OPRT polyclonal antibody. OPRT was positively stained in $54(54.5 \%)$ of 99 pancreatic cancers. The post-surgical survival rate of the OPRT (+) pancreatic cancers was significantly higher than that of the OPRT (-) ones. In the OPRT (+) group, the survival rate of the patients, who received adjuvant chemotherapy (ACT) with UFT, CPA or GEM, was significantly higher than that of the patients without ACT; however, in the OPRT (-) group, there was no difference in the survival between the ACT (+) and (-) groups. Multivariate analyses demonstrated that for all patients, primary tumor, status of nodal involvement $(\mathrm{pN})$,
\end{abstract}

Correspondence to: Dr Yoshinori Nio, ${ }^{4}$ Present address: Kodama Breast Clinic, 35-Kitano-kamihakubai-cho, Kita-ku, Kyoto 6038325, Japan

E-mail: kodama-breast-clinic@theia.ocn.ne.jp

Abbreviations: ACT, adjuvant chemotherapy; CPA, cyclophosphamide; DPD, dihydropyrimidine dehydrogenase; 5-FU, 5-fluorouracil; GEM, gemcitabine; OPRT, orotate phosphoribosyl transferase; PBS, phosphate-buffered saline; RT, radiotherapy; SA, surgery alone; UFT, uracil and tegafur; pAb, polyclonal antibody; pT, primary tumor; $\mathrm{pN}$, nodal involvement

Key words: pancreatic cancer, orotate phosphoribosyl transferase, UFT, chemotherapy, cyclophosphamide residual tumor, level of dissection and CPA were significant variables for the prognosis: in OPRT (+) groups, primary tumor, nodal involvement, GEM and CPA were significant variables. In contrast, in the OPRT (-) group, pN was the only significant variable. The present study is the first report on the significance of OPRT in human pancreatic cancer, and the results indicate that the expression of OPRT may be useful to predict the response to adjuvant chemotherapy in human pancreatic cancer.

\section{Introduction}

5-Fluorouracil (5-FU), an antitumor agent, is used clinically against a variety of malignancies. Orotate phosphoribosyl transferase (OPRT) is involved in the metabolism of the anticancer drug 5-fluorouracil (5-FU). 5-FU is a prodrug, and OPRT is the principal enzyme that converts 5-FU directly into an active antitumor metabolite, 5-fluoro-2'-deoxyuridine 5'-monophosphate. Accordingly, a high level of OPRT activity in tumors is thought to be a predictor of the 5-FU-sensitivity of the tumor. This has been supported by reports indicating that the expression of the OPRT gene was useful as a predictive parameter for the efficacy of fluoropyrimidine-based chemotherapy for various malignancies such as colorectal cancer $(1,2)$ and gastric cancer $(3)$.

On the other hand, OPRT is the key enzyme in the de novo DNA and RNA synthetic process, which suggests that OPRT may reflect the clinicopathological background of malignant diseases. In fact, it was reported that OPRT activity in primary lesion was a powerful predictor of nodal involvement in resectable gastric cancer (4). Furthermore, the OPRT mRNA levels were significantly higher in liver metastases than in primary colorectal cancer (5). In urologic malignancies, OPRT activity levels were correlated positively with the stage and grade of bladder and renal carcinomas $(6,7)$. In prostatic cancer, the OPRT expression level was significantly higher in carcinoma samples than in benign prostatic hypertrophy (8).

Thus, the significance of OPRT in various malignancies has been gradually clarified, although, to the authors' knowledge, little is known regarding the significance of OPRT in human pancreatic cancer. The present study was designed to assess the association between the activity of OPRT in tumors, and the clinicopathological status and prognosis of human 
Table I. OPRT expression and clinicopathologic characteristics.

\begin{tabular}{|c|c|c|c|c|}
\hline \multirow[b]{2}{*}{ Feature } & \multirow[b]{2}{*}{ No. of patients } & \multicolumn{2}{|c|}{ No. $(\%)$ of OPRT } & \multirow[b]{2}{*}{ Statistics } \\
\hline & & $(+)$ & $(-)$ & \\
\hline Overall & 99 & $54(54.5)$ & $45(45.5)$ & \\
\hline Age (years): mean & $65.3 \pm 9.3$ & $65.9 \pm 9.2$ & $64.5 \pm 9.4$ & $\mathrm{NS}^{\mathrm{b}}$ \\
\hline \multicolumn{5}{|l|}{ Gender } \\
\hline Male & 49 & $24(44.4)$ & $25(55.6)$ & \multirow[t]{2}{*}{ NS } \\
\hline Female & 50 & $30(55.6)$ & $20(44.4)$ & \\
\hline \multicolumn{5}{|l|}{ Surgery ${ }^{\mathrm{a}}$} \\
\hline PD or PPPD & 40 & $19(35.2)$ & $21(46.7)$ & \multirow[t]{3}{*}{ NS } \\
\hline DP & 45 & $30(55.5)$ & $15(33.3)$ & \\
\hline $\mathrm{TP}$ & 14 & $5 \quad(9.3)$ & $9(20.0)$ & \\
\hline \multicolumn{5}{|l|}{ Grade } \\
\hline 1 (well differentiated) & 20 & $11(20.4)$ & $9(20.0)$ & \multirow[t]{4}{*}{ NS } \\
\hline 2 (moderately differentiated) & 67 & $37(68.5)$ & $30(66.7)$ & \\
\hline 3 (poorly differentiated) & 12 & $6(11.1)$ & $6(13.3)$ & \\
\hline 4 (undifferentiated) & 0 & - & - & \\
\hline \multicolumn{5}{|l|}{ UICC pTNM stage } \\
\hline I & 9 & $7(13.0)$ & $2 \quad(4.4)$ & \multirow[t]{4}{*}{ NS } \\
\hline II & 12 & $7(13.0)$ & $5(11.1)$ & \\
\hline III & 43 & $21(38.9)$ & $22(48.9)$ & \\
\hline IV & 35 & $19(35.1)$ & $16(35.6)$ & \\
\hline \multicolumn{5}{|l|}{ pT (primary tumor) } \\
\hline 1 & 5 & $4 \quad(7.4)$ & $1 \quad(2.2)$ & \multirow[t]{4}{*}{ NS } \\
\hline 2 & 25 & $18(33.3)$ & 7 (15.6) & \\
\hline 3 & 40 & $16(29.6)$ & $24(53.3)$ & \\
\hline 4 & 29 & $16(29.6)$ & $13(28.9)$ & \\
\hline \multicolumn{5}{|l|}{ Nodal involvement } \\
\hline$(-)$ & 29 & $18(33.3)$ & $11(24.4)$ & \multirow[t]{2}{*}{ NS } \\
\hline$(+)$ & 70 & $36(66.7)$ & 34 (75.6) & \\
\hline \multicolumn{5}{|l|}{ Distant metastasis } \\
\hline$(-)$ & 91 & $50(92.6)$ & $41(91.1)$ & \multirow[t]{2}{*}{ NS } \\
\hline$(+)$ & 8 & $4 \quad(7.4)$ & $4 \quad(8.9)$ & \\
\hline
\end{tabular}

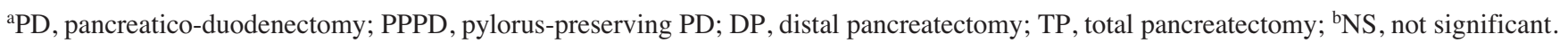

resectable pancreatic cancer, especially regarding its relevance to the efficacy of adjuvant chemotherapy with uracil and tegafur (UFT), cyclophosphamide (CPA) and/or gemcitabine (GEM). Here, we used immunohistochemical staining to evaluate the protein expression, because recently an anti-OPRT antibody has been developed (9). The immunohistochemical detection of tumoral OPRT protein expression may be useful for predicting the clinical response to 5-FU-based chemotherapy. To our knowledge, this is the first report evaluating the significance of OPRT in human pancreatic cancer.

\section{Materials and methods}

Patients. We obtained informed consent for this study on the genetic and histopathological background of the patients from the patients or their families according to the recommendation by the ethics committee of our department in 1999 .

Ninety-nine patients (50 females and 49 males; $35-80$ years old; mean 65.3 years) with pancreatic cancer underwent pancreatectomies between 1982 and 2005 at the First Department of Surgery, Shimane University School of Medicine. The present study included only invasive ductal carcinomas, but did not include mucinous cystic adenocarcinomas or intraductal papillary mucinous neoplasms with adenocarcinoma, because they have a better prognosis than invasive ductal carcinomas. The patients' profiles are summarized in Table I. A standard or pylorus-preserving pancreatoduodenectomy was performed in 40 patients, a distal pancreatectomy in 45 , and a total pancreatectomy in 14 patients. The tumors were staged according to the UICC classification (TNM classification) 
A

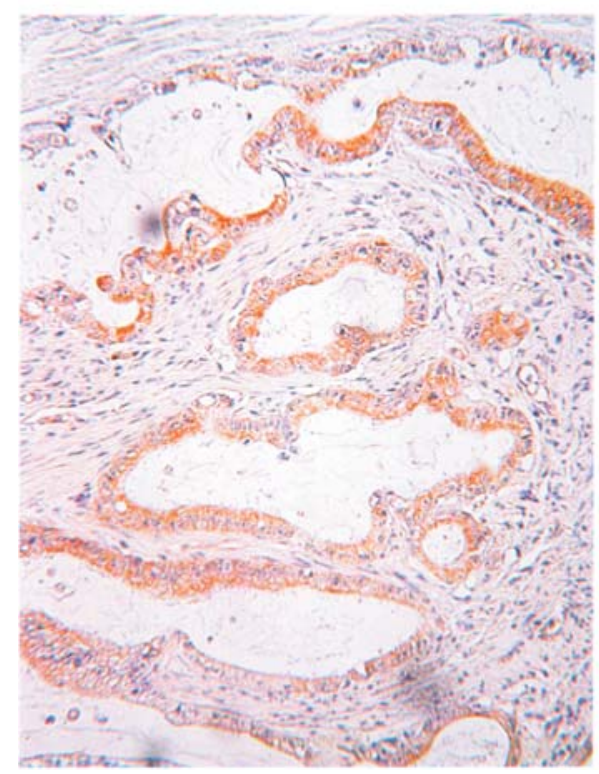

B

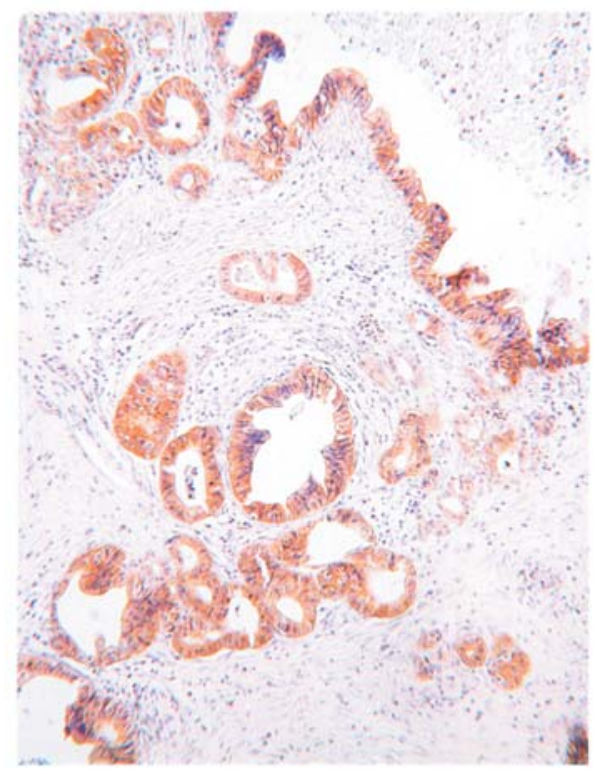

Figure 1. Representative immunostaining for OPRT. (A) Section from a 72-year-old woman with a moderately differentiated adenocarcinoma. (B) Section from a 76-year-old woman with a well-differentiated adenocarcinoma.

(10). Histopathologically, all specimens were verified to be IDCs of the pancreas. None of the patients received any type of treatment prior to their surgical procedures. After surgery, some patients were treated with adjuvant chemotherapy (ACT) and/or radiotherapy (RT), and were followed-up. All patients were followed-up in our department, and the survival of the patients was surveyed on October 1st, 2005. The post-operative survival was defined as the time that elapsed from the surgery to a cancer-related death.

Adjuvant chemotherapy (ACT). Of the 99 patients, 31 received surgery alone (SA), 63 received ACT and 5 received both ACT and RT. In Japan, under the universal health insurance system, the Japanese Ministry of Health, Labor and Welfare strictly regulates the use of anticancer agents. Accordingly, the ACT typically involves only approved agents. In our department, we have no standard regimen for ACT to treat pancreatic cancer, because there is no evidence supporting the survival benefits of adjuvant therapy for pancreatic cancer at present. Accordingly, the use of ACT was decided by the respective doctors with the informed consent of the patients and/or their families. Sixty-seven patients received ACT after surgery, and most patients were given 5-FU or its derivative UFT, alone $(n=25)$ or with CPA $(n=31)$, and some received intensive regimens including GEM $(n=8)$, adriamycin and cisplatin $(n=4)$. Five patients in the ACT group also received adjuvant RT using LINAC (ML-15MDX, 10MVX, Mitsubishi Electric Co. Ltd., Tokyo, Japan) at 50 Gy (2 Gy x 25) after surgery.

Antibody. The anti-OPRT rabbit polyclonal antibody (pAb) was supplied by Taiho Pharmaceutical Co. Ltd., Tokushima, Japan. It was reported that the protein level of OPRT measured by immunoblotting using this pAb closely correlated with the enzyme activity (9). This pAb was diluted to 1:500 for immunostaining (11).
Immunohistochemistry. The specimens were immunostained primarily according to the labeled polymer method using Dako EnVision $+^{\mathrm{TM}}$, Peroxidase, Rabbit kit (Dako Corp., Carpinteria, CA, USA), which is a goat anti-rabbit immunoglobulin conjugated to a peroxidase labeled-dextran polymer. Formalinfixed, paraffin-embedded specimens were cut into 4- $\mu \mathrm{m}$ sections and floated briefly on warm water for paraffin section stretching. The temperature of warm water is strictly applied below $45^{\circ} \mathrm{C}$ for preserving the antigenicity in paraffin sections (11). Finally, the sections were mounted on silanized glass slides (S3003 Dako) and dried in an oven at $60^{\circ} \mathrm{C}$ for several hours. The sections were deparaffinized using a routine procedure. Endogenous peroxidase activity was blocked by treatment with $3 \%$ hydrogen peroxide for $15 \mathrm{~min}$. The specimens were incubated with the primary $\mathrm{Ab}$ for $1 \mathrm{~h}$ at room temperature, and then rinsed three times in phosphate-buffered saline (PBS). The specimens were incubated with EnVision+, Peroxidase, Rabbit at room temperature for $30 \mathrm{~min}$, and then rinsed three times in PBS. Finally, the specimens were treated with a $0.05 \% 3,3^{\prime}$-diaminobenzidine solution for $10 \mathrm{~min}$ at room temperature. Nuclei were counterstained with Mayer's hematoxylin.

Evaluation of immunostaining. The immunostaining was considered positive for OPRT only when cytoplasmic immunoreactivity in tumor cells was diffusely observed. Those cases with only faint immunostaining were regarded as negative.

Statistical analysis. The correlations between the clinicopathological factors and the expression of OPRT were examined using Pearson's correlation analysis. The post-surgical status of all patients was surveyed on January 1st, 2006. The cumulative survival rates were calculated according to the Kaplan-Meier method, and were compared by the Cox-Mantel test. A multivariate analysis of Cox's proportional hazard risk 


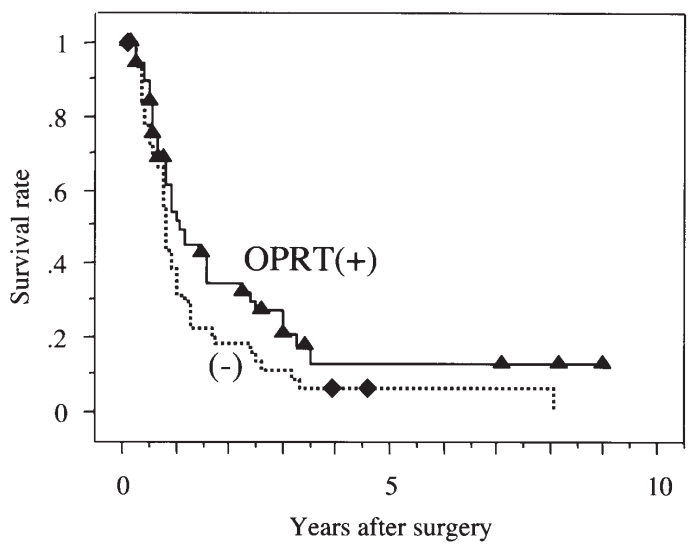

Figure 2. OPRT expression and survival curves. OPRT $(+), \mathrm{n}=54$; OPRT (-), $\mathrm{n}=45$. OPRT $(+)$ vs. OPRT $(-), \mathrm{p}=0.0312$.

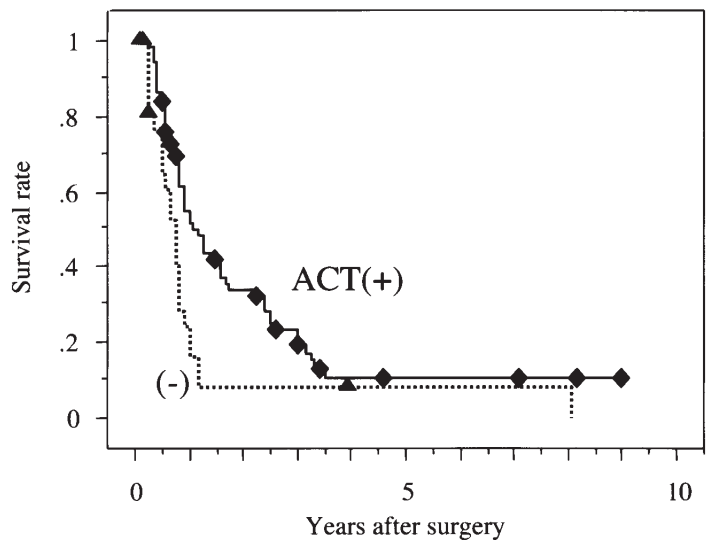

Figure 3. Effect of adjuvant chemotherapy (ACT) on patient survival. ACT $(+), n=68 ; \operatorname{ACT}(-), n=31$. ACT (+) vs. ACT (-), p=0.0027.
A

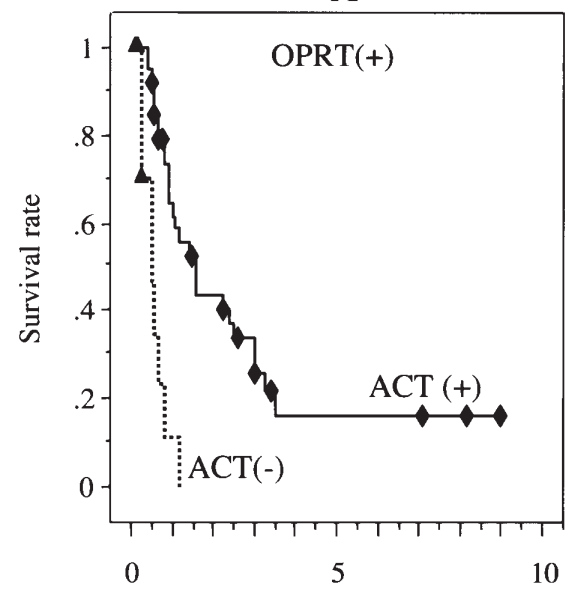

B

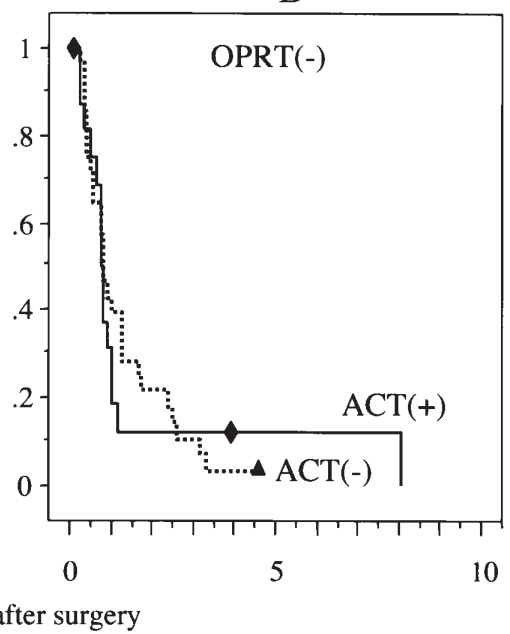

Figure 4. Effect of OPRT expression on the efficacy of adjuvant chemotherapy (ACT). (A) OPRT (+) pancreatic cancer: ACT (+) (n=40) vs. ACT (-) (n=14), $\mathrm{p}<0.0001$. (B) OPRT (-) pancreatic cancer: ACT (+) (n=28) vs. ACT (-) $(\mathrm{n}=17), \mathrm{p}=0.8248$.

model was used to obtain the conditional risk of death due to IDC of the pancreas. Statistically significant differences were defined at $\mathrm{p}<0.05$.

\section{Results}

A representative expression of OPRT is demonstrated in Fig. 1. The OPRT was stained in the cytoplasm of the cells. OPRT was positively stained in $54(54.5 \%)$ of 99 pancreatic cancers (Table I). The expression of OPRT did not correlate with any clinicopathological factors for pancreatic cancer, such as age, gender, stage or histological grade (Table I). The postsurgical survival rate of the patients with OPRT (+) pancreatic cancer was higher than that of those with OPRT (-) pancreatic cancer ( $p=0.0312$ ) (Fig. 2). The ACT with UFT, CPA or GEM was effective to improve the survival after surgery, and the survival curve of the ACT group was significantly higher than that of the ACT (-) group ( $\mathrm{p}=0.0027)$ (Fig. 3). The OPRT expression was found to have a significant effect on the efficacy of the ACT, and in the OPRT (+) group, the survival rate of the patients, who received ACT, was significantly higher than that of the patients without ACT $(\mathrm{p}<0.0001)$ (Fig. 4A). However, in the OPRT (-) group, there was no difference in the survival between the ACT (+) and (-) groups (Fig. 4B).

Since the present study was not a randomized controlled study, a multivariate analysis was applied to evaluate the significance of clinicopathological factors on the survival of the patients after surgery. Multivariate analyses demonstrated that in overall patients, the primary tumor $(\mathrm{pT})(\mathrm{p}=0.0145)$ and status of nodal involvement $(\mathrm{pN})(\mathrm{p}=0.0001)$ were significant variables increasing the risk to prognosis and residual tumor $(\mathrm{p}=0.0607)$ was a variable increasing the risk with borderline significance. In contrast, the level of dissection $(\mathrm{p}=0.0020)$ and CPA $(\mathrm{p}=0.0272)$ were significant variables decreasing the risk to prognosis and UFT ( $\mathrm{p}=0.0985$ ) was a variable decreasing the risk with borderline significance. However, GEM ( $\mathrm{p}=0.0635$ ) was a risk-increasing variable with borderline significance to the prognosis (Table II). Subset analysis demonstrated that in OPRT $(+)$ groups, $\mathrm{pN}(\mathrm{p}=0.0008)$ and GEM $(p=0.0110)$ were significant variables increasing the risk to prognosis, but $\mathrm{CPA}(\mathrm{p}=0.0015)$ was a significant 
Table II. Multivariate analysis in overall patients.

\begin{tabular}{lcc}
\hline Variable & $\begin{array}{c}\text { Risk ratio } \\
(95 \% \text { confidence })\end{array}$ & P-value \\
\hline Age & $0.989(0.963-1.016)$ & 0.4140 \\
Gender (male, $1 /$ female, 0$)$ & $0.905(0.544-1.506)$ & 0.7005 \\
Histological grade $(1 / 2 / 3 / 4)$ & $1.276(0.779-2.092)$ & 0.3330 \\
pT $(1 / 2 / 3 / 4)$ & $1.498(1.084-2.071)$ & 0.0145 \\
pN $(0 / 1)$ & $3.343(1.792-6.236)$ & 0.0001 \\
M $(0 / 1)$ & $1.729(0.682-4.387)$ & 0.2488 \\
Dissection level $(0 / 1 / 2)$ & $0.551(0.378-0.803)$ & 0.0020 \\
Residual tumor $(0 / 1 / 2)$ & $1.527(0.981-2.377)$ & 0.0607 \\
OPRT expression $(0 / 1)$ & $0.905(0.607-1.350)$ & 0.6242 \\
UFT $(0 / 1)$ & $0.584(0.308-1.105)$ & 0.0985 \\
CPA $(0 / 1)$ & $0.405(0.181-0.903)$ & 0.0272 \\
GEM $(0 / 1)$ & $2.382(0.952-5.961)$ & 0.0635 \\
Other chemotherapy $(0 / 1)$ & $1.027(0.466-2.262)$ & 0.9477 \\
Radiotherapy $(0 / 1)$ & $1.258(0.505-3.131)$ & 0.6219 \\
\hline
\end{tabular}

variable decreasing the risk, while UFT was a variable decreasing the risk with borderline significance. In contrast, in the OPRT (-) group, pN (0.0492) was the only significant variable and the level of dissection was a risk-decreasing variable with borderline significance (Table III).

\section{Discussion}

OPRT is one of the key enzymes involved in the metabolism of fluoropyrimidines, which converts 5-FU to its active form. Accordingly, OPRT activity in tumors is thought to reflect the efficacy of various fluoropyrimidines, as well as the prognosis of malignant diseases. In fact, recent reports demonstrated that
OPRT is associated with the efficacy of fluoropyrimidine-based chemotherapy in various malignancies, such as colorectal, gastric and lung cancers. However, to our knowledge, there have been no reports on the role of OPRT in pancreatic cancer. Accordingly, the present study may be the first such report.

The present study used immunohistochemical methods to evaluate the protein expression of OPRT. This method is not a quantitative analysis, but is simple and easy and can evaluate the expression of OPRT in paraffin-embedded specimens. Therefore, immunohistochemical detection of tumoral OPRT protein expression with anti-OPRT pAb may provide a useful method for predicting the clinical response to 5-FU-based chemotherapy.

In the present study, the expression of OPRT was a beneficial predictor for UFT-based ACT, and in patients with OPRT (+) pancreatic cancer, the survival curve of the patients who received oral UFT-based ACT, was significantly higher than that of the surgery alone group. This result coincides with previous reports, and several reports indicated that expression of the OPRT gene was useful to predict the efficacy of fluoropyrimidine-based chemotherapy for various malignancies. In metastatic colorectal cancer, the mRNA expression of OPRT, which was measured using real-time quantitative RT-PCR assays of surgically resected materials from primary colorectal tumors, was very useful for predicting the effects of 5-FUbased hepatic artery injection chemotherapy for synchronous liver metastases in 22 patients (2). It was also reported that in 37 patients with metastatic colorectal cancer, who received oral treatment of uracil and tegafur (UFT) and leucovorin, the patients with high OPRT gene expression survived longer than those with low OPRT expression, and the authors emphasized that both expression of the OPRT gene and the OPRT/DPD ratio might be useful as predictive parameters for the efficacy of fluoropyrimidine-based chemotherapy for metastatic colorectal cancer (1). In gastric cancer, it was reported that the combination expression of OPRT and TS in prechemotherapeutic fresh frozen samples obtained from

Table III. Multivariate analysis grouped by OPRT expression.

\begin{tabular}{|c|c|c|c|c|}
\hline \multirow[b]{2}{*}{ Variable } & \multicolumn{2}{|l|}{ OPRT (+) } & \multicolumn{2}{|l|}{ OPRT (-) } \\
\hline & Risk ratio ( $95 \%$ confidence) & P-value & Risk ratio (95\% confidence) & P-value \\
\hline Age & $1.002(0.967-1.039)$ & 0.9044 & $0.989(0.934-1.047)$ & 0.7045 \\
\hline Gender (male, $1 /$ female, 0 ) & $0.556(0.225-1.377)$ & 0.2048 & $0.983(0.416-2.326)$ & 0.9698 \\
\hline Histological grade $(1 / 2 / 3 / 4)$ & $2.012(0.679-5.966)$ & 0.2074 & $1.161(0.608-2.216)$ & 0.6503 \\
\hline pT $(1 / 2 / 3 / 4)$ & $1.577(0.969-2.564)$ & 0.0665 & $1.301(0.763-2.221)$ & 0.3339 \\
\hline $\mathrm{pN}(0 / \mathrm{l})$ & $5.535(2.040-15.014)$ & 0.0008 & $2.760(1.003-7.596)$ & 0.0492 \\
\hline $\mathrm{M}(0 / 1)$ & $1.592(0.426-5.949)$ & 0.4895 & $1.657(0.277-9.913)$ & 0.5803 \\
\hline Dissection level $(0 / 1 / 2)$ & $0.642(0.355-1.159)$ & 0.1416 & $0.568(0.309-1.043)$ & 0.0680 \\
\hline Residual tumor $(0 / 1 / 2)$ & $2.011(0.940-4.304)$ & 0.0717 & $1.725(0.785-3.792)$ & 0.1748 \\
\hline UFT $(0 / 1)$ & $0.306(0.086-1.083)$ & 0.0663 & $0.715(0.305-1.675)$ & 0.4394 \\
\hline $\mathrm{CPA}(0 / 1)$ & $0.143(0.043-0.474)$ & 0.0015 & $0.671(0.156-2.887)$ & 0.5924 \\
\hline $\operatorname{GEM}(0 / 1)$ & $4.896(1.439-16.659)$ & 0.0110 & $0.147(0.010-2.245)$ & 0.1679 \\
\hline Other chemotherapy $(\mathrm{O} / 1)$ & $2.853(0.525-15.500)$ & 0.2246 & $1.126(0.389-3.259)$ & 0.8269 \\
\hline Radiotherapy (0/1) & $2.182(0.687-6.924)$ & 0.1856 & $9.782(0.567-168.795)$ & 0.1165 \\
\hline
\end{tabular}


primary tumors might predict the response to S-1 in 59 metastatic gastric cancer patients as a first line treatment (3). In resectable non-small cell lung cancers, the 5-year survival rate of patients with OPRT (+) stage II-III tumors was significantly higher than that of those with OPRT (-) stage IIIII tumors (12).

The present study indicated that CPA was a significant variable to decrease risk, especially in OPRT (+) pancreatic cancers, and that CPA is linked to the efficacy of fluoropyrimidines or that the effects of CPA are affected by the OPRT. We previously demonstrated that UFT administration increased the level of DPD activity, but that CPA inhibited the DPD level, in breast cancer (13). Accordingly, the present results suggest that CPA may affect the activities of DPD as well as various enzymes associated with 5-FU metabolism.

Another interesting result was that GEM was a significant variable increasing the risk to prognosis of OPRT $(+)$ pancreatic cancer. In OPRT (-) pancreatic cancer, GEM is a variable of decreasing risk with no statistical significance $(p=0.1679)$. GEM is also a pyrimidine-antagonist like 5-FU. Cytidine deaminase, 5'-nucleotidase, deoxycytidine kinase and MRP5 are thought to be related to the effects of GEM. Therefore, OPRT may not be associated with the efficacy of GEM, but it is not so surprising that OPRT expression is associated with expression of these GEM-associated enzymes. The present results suggest that OPRT (-) may be a useful predictor of response to GEM. However, in the present study only eight patients received GEM, and further study is necessary to reach a conclusive result.

Recently, a combination regimen with docetaxel and S-1 for advanced or recurrent gastric cancer was reported to result in a $56.2 \%$ objective response (14), and they reported that this combination produced an increased level of OPRT activity and a decreased level of DPD, suggesting that biochemical modulation of the two drugs had occurred (15), Accordingly, biochemical modulation will be expanded from the viewpoint of OPRT augmentation as well as inhibition of TS and DPD.

Finally, there was an interesting report that OPRT Gly213Ala polymorphism seems to be a useful marker for predicting toxicity to bolus 5-FU chemotherapy (16). It is well known that a DPD deficiency is associated with severe toxicity after the administration of 5-FU or its derivatives (17). Accordingly, OPRT is more extensively studied with regard to its antitumor effects as well as toxicities of fluoropyrimidines.

These results suggest that the expression of OPRT may be a useful factor to predict the response to adjuvant chemotherapy in human pancreatic cancer.

\section{Acknowledgements}

We thank Ms. Miyuki Ishihara, Ms. Yasuko Sonoyama and Ms. Yuka Ouchi for their excellent assistance. Part of this study was supported by a Grant-in-Aid for Scientific Research (C) (no.17591400) from Japan Society for the Promotion of Science (JSPS).

\section{References}

1. Ichikawa W, Uetake H, Shirota Y, et al: Both gene expression for orotate phosphoribosyltransferase and its ratio to dihydropyrimidine dehydrogenase influence outcome following fluoropyrimidine-based chemotherapy for metastatic colorectal cancer. Br J Cancer 89: 1486-1492, 2003.

2. Matsuyama R, Togo S, Shimizu D, et al: Predicting 5-fluorouracil chemosensitivity of liver metastases from colorectal cancer using primary tumor specimens: three-gene expression model predicts clinical response. Int J Cancer 119: 406-413, 2006.

3. Ichikawa W, Takahashi T, Suto K, et al: Simple combinations of 5-FU pathway genes predict the outcome of metastatic gastric cancer patients treated by S-1. Int J Cancer 119: 1927-1933, 2006.

4. Ochiai T, Sugitani M, Nishimura K, et al: Impact of orotate phosphoribosyl transferase activity as a predictor of lymph node metastasis in gastric cancer. Oncol Rep 14: 987-992, 2005.

5. Inokuchi M, Uetake H, Shirota Y, et al: Gene expression of 5fluorouracil metabolic enzymes in primary colorectal cancer and corresponding liver metastasis. Cancer Chemother Pharmacol 53: 391-396, 2004.

6. Mizutani Y, Wada H, Fukushima M, et al: Prognostic significance of orotate phosphoribosyltransferase activity in bladder carcinoma. Cancer 100: 723-731, 2004.

7. Mizutani Y, Wada H, Yoshida O, et al: Significance of orotate phosphoribosyl transferase activity in renal cell carcinoma. J Urol 171: 605-610, 2004.

8. Miyoshi Y, Uemura $\mathrm{H}$, Ishiguro $\mathrm{H}$, et al: Expression of thymidylate synthase, dihydropyrimidine dehydrogenase, thymidine phosphorylase, and orotate phosphoribosyl transferase in prostate cancer. Prostate Cancer Prostatic Dis 8: 260-265, 2005.

9. Sakamoto K, Sugimoto Y, Miyadera K, et al: Preparation of anti-orotate phosphoribosyltransferase antibody and its application to immunochemical detection in human tumor cells. Int J Mol Med 16: 245-249, 2005.

10. TNM classification of malignant tumours. 6th edition. International Union Against Cancer. A John Wiley \& Sons, Inc., Publication, 2002.

11. Kamoshida S, Sakamoto N, Matsuoka H, et al: Heat-assisted stretching of paraffin sections on hot plate weakens immunoreactivity of orotate phosphoribosyltransferase. Acta Histochem Cytochem 38: 69-74, 2005.

12. Nakano J, Huang C, Liu D, et al: Evaluations of biomarkers associated with 5-FU sensitivity for non-small-cell lung cancer patients post-operatively treated with UFT. Br J Cancer 95: 607-615, 2006.

13. Nio Y, Iguchi C, Kodama H, et al: Cyclophosphamide augments the anti-tumor efficacy of uracil and tegafur by inhibiting dihydropyrimidine dehydrogenase. Oncol Rep 17: 153-159, 2007.

14. Yoshida K, Ninomiya M, Takakura N, et al: Phase II study of docetaxel and S-1 combination therapy for advanced or recurrent gastric cancer. Clin Cancer Res 12: 3402-3407, 2006.

15. Wada Y, Yoshida K, Suzuki T, et al: Synergistic effects of docetaxel and S-1 by modulating the expression of metabolic enzymes of 5-fluorouracil in human gastric cancer cell lines. Int J Cancer 119: 783-791, 2006.

16. Ichikawa W, Takahashi T, Suto K, et al: Orotate phosphoribosyltransferase gene polymorphism predicts toxicity in patients treated with bolus 5-fluorouracil regimen. Clin Cancer Res 12: 3928-3934, 2006.

17. Van Kuilenburg AB: Dihydropyrimidine dehydrogenase and the efficacy and toxicity of 5-fluorouracil. Eur J Cancer 40: 939-950, 2004. 